\title{
Recent Advances in Treatment for Uremic Pruritus
}

\author{
Hiromichi Suzuki ${ }^{*}$, Hiroshi Omata ${ }^{2}$, Hiroo Kumagai ${ }^{3}$ \\ ${ }^{1}$ Department of Nephrology, Saitama Medical University, Moroyama, Japan \\ ${ }^{2}$ Center for Oriental and Integrated Medicine, Saitama Medical University, Moroyama, Japan \\ ${ }^{3}$ Department of Nephrology and Endocrinology, National Defense Medical College, Tokorozawa, Japan \\ Email: ${ }^{*}$ iromichi@saitama-med.ac.jp
}

Received 23 February 2015; accepted 8 March 2015; published 13 March 2015

Copyright (C) 2015 by authors and Scientific Research Publishing Inc.

This work is licensed under the Creative Commons Attribution International License (CC BY). http://creativecommons.org/licenses/by/4.0/

(c) (i) Open Access

\begin{abstract}
This review highlights recent advances in pathophysiology and treatment for uremic pruritus, especially focusing on various interventions. Pruritus in patients on hemodialysis (HD) and peritoneal dialysis (PD) still remains an unresolved issue. Recently, the efficacy and safety of nalfurafine hydrochloride have been reported in Japan, and at present, more than thirty thousand patients receive this new drug. In comparison with the efficacy of this new drug, acupuncture, a form of traditional Japanese therapy, has been validated for relief of symptoms of pruritus. In this review, various interventions for relief of symptoms of pruritus as well as recent studies on its pathophysiology will be introduced. This review will be helpful for treatment of pruritus in patients on dialyzed therapy in clinical practice.
\end{abstract}

\section{Keywords}

Itch, Dialysis, Ultraviolet, Topical Ointment, Acupuncture, Opioid Antagonists, Nalfurafine

\section{Introduction}

Uremic pruritus remains a frequent concern for hemodialysis patients with the most frustrating and disabling symptoms. Nearly $90 \%$ of patients on dialysis suffer from pruritus. Until present, there have been a lot of reviews discussing the pathophysiology and treatment of pruritus [1]-[14]. Previously, the word "uremic pruritus" has been used for symptoms of itching because it is a common skin derangement in patients with advanced renal failure. However, the usage of "uremic" may cause confusion because pruritus is not found in patients with acute kidney injury. In this regard, Paitel et al. [9] recently proposed the term, "chronic kidney disease (CKD)-asso-

${ }^{*}$ Corresponding author. 
ciated pruritus" instead of "uremic pruritus" as a more precise nomenclature. In this review, the words "uremic pruritus”, “CKD-associated pruritus” and "pruritus” are used interchangeably because the authors would like to respect each author's contribution. The prevalence of CKD-associated pruritus was found to range from $15 \%$ 90\% of patients [15] [16]. Recent data from the Dialysis Outcomes and Practice Patterns Study (DOPPS) reported that the prevalence of CKD-associated pruritus was 42\% [17].

\section{Clinical Characteristics of CKD-Associated Pruritus}

In patients with CKD, skin lesions are usually not found. Generally, skin lesions are secondary changes such as excoriations with or without impetigo, linear crusts, papules, and ulcerations. Half of patients have generalized itching, and in the other half, pruritus is localized to the back, limbs, chest or head. Pruritus is intermittent or prolonged over hours and days, and becomes worse at night [18] [19]. Mettang \& Krener [14] stated in their review that the diagnosis of uremic pruritus may be challenging because many patients with end-stage renal disease (ESRD) are suffering from other diseases, such as cardiovascular diseases, diabetes mellitus, chronic liver or hematological diseases, which may provoke itching either by itself or by medication given to treat these entities.

\section{Why Is Pruritus Problematic?}

A recent longitudinal study of CKD-associated pruritus in HD patients have clearly demonstrated that significant associations were found among itching intensity, severity, and health-related quality of life (HR-QOL) measures in domains such as mood, social relations, and sleep. In Japan, Narita et al. [16] recruited a total of 1773 patients on HD and evaluated the severity of pruritus with a visual analogue scale (VAS). Four hundred and fifty-three patients had severe pruritus with a VAS score of more than or equal to 7.0. Further, 70\% of these patients complained of sleep disturbances. From these data, it is clear that patients who suffer from pruritus also have a lower HR-QOL including sleep disturbances which may lead to poor prognosis.

\section{Pathogenesis of Itch}

The exact pathophysiological mechanisms of CKD-associated pruritus still remain unexplained. Recently, the role of opioid $\mu$-receptors in the central nervous system and skin has been focused on as a promising candidate in the pathophysiological mechanism of itch [20]. Further, a more recent report showed an imbalance between the antagonistic activities of $\mu$ - and $\kappa$-opioid receptors in favor of $\mu$-receptor activation in CKD-associated pruritus [21].

In conjunction with other advances in the pathophysiology of itch, some central imaging studies on itch using positron emission tomography (PET) and functional magnetic resonance image (fMRI) have provided further information on itch processing in the brain [22]. In these studies, the premotor areas, prefrontal cortex, anterior cingulate cortex, and cerebellum were found to be activated. When comparing the brain areas involved in itch and pain processing, a large overlap between the two sensations was identified. However, this comparison is based on only a few studies and is largely regarded as preliminary.

The itch-selective spinal neurons from a distinct pathway project from lamina I of the spinal cord to the ventrocaudal part of the nucleus medialis, which projects to the anterior cingulate and dorsal insular cortices. The supraspinal processing of itch and its corresponding scratch response in humans have recently been investigated by PET and fMRI. Induction of itch by histamine application coactivates the anterior cingulate and insular cortices, premotor and supplementary motor areas, cerebellum, primary somatosenstory cortex, and thalamus. As done earlier for pain sensation, particular aspects of the itch sensation are correlated with the activation of certain brain areas; spatial and temporal aspects may be processed in the primary somatosensory cortex, planning of the scratch response in the premotor and supplementary motor cortices, and affective and motivational aspects in the anterior cingulate and insular cortices.

In addition, slow-conducting c-fibers that transduce itch spinal have recently been discovered [23], and these new findings will develop further our understanding of the neurobiology of itch.

\section{Treatment for CKD-Associated Pruritus}

\subsection{Modification of Dialysis Techniques}

Introduction of the use of biocompatible dialysis membranes has reduced the prevalence of pruritus in HD pa- 
tients [7]. However, it still remains uncertain whether alterations in dialysis therapy including changes in dialysis membrane can reduce pruritus [24] [25] or not [26]. Hiroshige et al. [27] analyzed data on 59 HD patients, who did not have disorders in calcium and phosphate metabolism, and found that more than $60 \%$ of them suffered from disabling pruritus possibly related to chronic uremia. Blood urea nitrogen (BUN) and plasma $\beta_{2}-$ microglobulin, both of which are biochemical factors that are associated with the prevalence of pruritus and dialysis efficacy, were investigated and calculated by urea kinetics. Significantly higher values of BUN and plasma $\beta_{2}$-microglobulin were observed just before the dialysis session in pruritic patients with lower dialysis efficacy as estimated by Kt/V urea and normalized protein catabolic rate (nPCR), respectively. After 3 months without changing the dialysis prescriptions, 16 patients with a mean Kt/V urea and an nPCR of 1.28 and 1.22 $\mathrm{g} / \mathrm{kg} /$ day, respectively, experienced significant reductions in the degree of pruritus as estimated by the pruritic score, from $12.6 \pm 5.1$ to $6.3 \pm 3.2(P<0.001)$. Twenty-two patients with a mean Kt/V urea and an nPCR of 1.09 and 1.01, respectively, continued to suffer severe pruritus (score: $12.3 \pm 4.7$ to $12.7 \pm 6.4, P=$ NS). In 9 of 22 patients with prolonged severe pruritus, dialysis efficacy was heightened with an increase in dialyzer membrane area of more than $0.3 \mathrm{~m}^{2}$. Seven of 9 patients with increased dialysis prescription had significant reductions in the mean pruritic score, from $12.6 \pm 4.8$ to $6.3 \pm 2.4$, which was inversely related to the significant increase of $\mathrm{Kt} / \mathrm{V}$ urea from $1.05 \pm 0.25$ to $1.24 \pm 0.33(P<0.05)$; among patients whose dialysis prescription did not change, only one had a significant reduction in pruritic score. The authors concluded that higher dialysis efficacy with good nutritional state reduces the prevalence and degree of pruritus in HD patients.

Previously, Graf et al. [28] reported that lowering the dialysate magnesium concentration can restore nerve conduction velocity towards normal in patients receiving HD, and this could be the reason for the complete disappearance of pruritus in the study by Hiroshige et al. [24]. In contrast, Carmichael et al. [29] failed to demonstrate a beneficial effect of reduction in magnesium on pruritus. In their trial, although they showed that a magnesium-free dialysis fluid corrected hypermagnesaemia, it failed to improve renal itch. In addition, the fall in serum magnesium concentration was associated with an increased concentration of parathyroid hormone, as previously noted, with the potential of producing renal osteodystrophy in the long term. It is therefore difficult to generalize the findings from the study by Hiroshige and colleagues.

The role of calcium in the dialysate was discussed by Kyriazi et al. [30], who showed that reduction in dialysis calcium concentrations from 1.75 to $1.0 \mathrm{mmol} / \mathrm{L}$ was associated with a $41.421 \% \pm 8.47 \%(P<0.05)$ relief from itching in 4 HD patients, indicating that at least in some uremic individuals, ionized calcium (iCa) has a pivotal role in the neuropathophysiology of CKD-associated pruritus. It has been postulated that calcium contributes to itching by influencing the degranulation of cutaneous mast cells, thus appearing to be a modifier rather than an initiator of CKD-associated pruritus.

Polymethylmethacrylate (PMMA) artificial kidney (AK) has been reported to adsorb more serum cytokines than other high-flux AK. In 30 patients with severe uremic pruritus out of 300 chronic patients in a single center who entered this prospective study, the dialyzers were changed to PMMA AK for 4 weeks. There were no significant differences in the laboratory assay results including predialysis serum BUN, creatinine $(\mathrm{Cr}), \beta_{2}$ microglobulin, calcium, phosphate, calcium-phosphate product, intact parathyroid hormone (iPTH), ferritin, hematocrit, high-sensitivity C-reactive protein (hsCRP), interleukin (IL)-1 $\beta$, IL-2, IL-6, IL-18, tumor necrosis factor (TNF)- $\alpha$, and Kt/V. PMMA AK was effective in reducing the pruritus score from $23.46 \pm 11.94$ to $7.38 \pm$ $6.42(P<0.001)$. The effect of uremic pruritus relief appeared after 1 week of PMMA AK use. In spite of this study, the mechanism for the beneficial effect of PMMA AK on uremic pruritus remains to be determined. However, it is proposed that PMMA AK may be a useful adjuvant therapy in chronic HD patients with severe uremic pruritus.

In line with this study, Kato et al. [31] conducted a 6-month prospective and crossover trial to investigate the effect of PMMA membrane for renal itching. They examined the role of the TNF- $\alpha$ system in pruritus in hemodialysis patients through assessment of the degree of skin itching and measurement of circulating levels of TNF- $\alpha$ and soluble TNF receptors (sTNFR-I, sTNFR-II) in 19 HD patients, who were complicated with prolonged severe pruritus for 6 months. However, no association was found between the degree of pruritus and circulating sTNFR-I and II values. Skin itching scale was significantly decreased from $2.7 \pm 0.2$ to $2.1 \pm 0.3$ following the use of PMMA membrane for 3 months $(P<0.05)$. In contrast, there was no change in itching scale during 3 months of conventional therapy ( $2.2 \pm 0.3$ versus $2.2 \pm 0.3, P=$ NS). PMMA itself did not affect serum TNF- $\alpha$ and STNFR values as compared to conventional dialyzer membranes. These findings suggested that the PMMA dialyzer can improve renal itching, but not through modification of the TNF- $\alpha$ system. 


\subsection{Topical Treatments}

Emollients have been shown to be beneficial in patients with CKD-associated pruritus [32]-[34]. In general, emollients are proposed for use as first-line treatment. Among emollients, aqueous gels have been shown to reduce pruritus; previously, Okada and Matsumoto [35] demonstrated that emollients with high water content effectively reduced itch. In their report, $20 \mathrm{HD}$ patients were divided into two groups; one group was treated with an aqueous gel containing 80\% water (ADJUPEX Ensemble gel, ADJUPEX Co. Ltd., Tokyo, Japan) and another group did not receive any emollient treatment. The aqueous gel consisted of $80 \mathrm{~g}$ of water and $20 \mathrm{~g}$ of aloe vera extract, silk powder, naturally-derived vitamin E, squalane, and other naturally-derived ingredients. The gel contained no synthetic surfactants, artificial fragrance, color mineral oil, synthetic antioxidants, or alcohol. The emollient was applied twice daily for 2 weeks. VAS scores for itching at 2 weeks were significantly decreased compared with that at week 0 . In addition, skin dryness was significantly improved. The results showed that an aqueous gel containing high water content effectively improves itching in HD patients with mild uremic pruritus. Besides, psychological discomfort also improved.

These findings corroborated those that were already reported by Itai et al. [36], who examined the effects of aromatherapy (odorless condition, lavender, and hiba oil) on mood and anxiety in 14 female patients who were being treated with chronic HD. A control period consisting of natural hospital smells was established before each test session, and then aromatic test conditions including lavender and hiba oil aromas were systematically compared with odorless conditions. The effects of aromatherapy were measured using the Hamilton rating scale for depression (HAMD) and the Hamilton rating scale for anxiety (HAMA). Hiba oil aroma significantly decreased the mean scores of HAMD and HAMA, and lavender aroma significantly decreased the mean scores of HAMA. The mean scores of HAMD and HAMA in odorless conditions were not significantly different from those of the control conditions. These results indicate that in chronic HD patients, hiba oil is an effective, non-invasive treatment for depression and anxiety.

In another study, a cream with structured physiological lipids (DMS, Derma Membrane Structure) and endogenous cannabinoids was tested for 3 weeks in 21 subjects with pruritus [34]. A significant reduction in pruritus was noted during the test product application using both scales for itching intensity assessment $(P<0.0001)$. Pruritus was significantly decreased at the end of the 3-week treatment $(P=0.02)$ as compared to before treatment, and was completely eliminated in eight patients (38.1\%). However, the symptoms worsened slightly 2 weeks after test discontinuation, although it was still significantly less intensive than before treatment $(P<$ 0.001). During the follow-up period, pruritus appeared again in 2 of these 8 subjects, but disappeared in an additional 2 patients. Therefore, at follow-up, 14 days after the end of the treatment, 8 patients (38.1\%) were still free from itch. Further, xerosis was also observed in all test subjects. A 3-week treatment period resulted in complete reduction of xerosis in 17 patients (81\%). During follow-up 14 days after the end of the therapy, symptoms of dry skin appeared again in 4 patients, while 13 patients (61.9\%) still had smooth skin. Xerosis scores were significantly reduced during the whole study period. After discontinuation of the trial, the scores for dry skin increased, although the difference between day 21 (end of the therapy) and follow-up visit did not reach statistical significance. At follow-up visit, xerosis remained significantly less intensive as compared to the beginning of the study. The test product was very well-tolerated by all patients (100\%). At the end of the treatment period, 11 patients (52.4\%) found the final result of the treatment satisfactory, 8 patients (38.1\%) considered it as very satisfactory, and only 2 patients (9.5\%) stated that the result of the therapy was unsatisfactory. This is considered the first study to evaluate topical application of a preparation containing endocannabinoids in the treatment of uremic pruritus. In this preliminary open study, they demonstrated that the preparation with structured natural lipids and endocannabinoids could be of help in controlling itching and xerosis in patients on maintenance HD. Based on the present knowledge, the exact mechanism of antipruritic action of the tested cream could only be hypothesized. Although the antipruritic action of the evaluated preparation could be related to the its moisturizing effect, it is very probable that the observed decrease in pruritus with this therapy was not only the result of the improvement in dry skin. Based on these findings, these effects could be influenced by additional active ingredients of endocannabinoids. Dvorak et al. [37] showed that cannabinoid receptor agonists significantly reduced histamine-induced itch and vasodilatation by topical application of them before administration of histamine. In addition, N-palmitoylethanolamine was demonstrated to down-modulate mast cell degranulation induced either by neurogenic- or immune-mediated stimuli [38].

Another naturally-derived agent that may be helpful in reducing pruritus is capsaicin, which is an alkaloid extracted from the common pepper plant and marketed as a topical analgesic. Capsaicin owes its potential antipru- 
ritic properties to desensitization of nociceptive nerve endings depleting the peripheral neurons of substance $\mathrm{P}$ and perhaps blocking the conductor of pain or pruritus. Moreover, it is highly likely that capsaicin preferentially activates nociceptive fibers and probably, by acting as an analgesic, indirectly affects itch inhibition. However, the painful burning sensation associated with capsaicin use frequently leads to treatment withdrawal.

Breneman et al. [39] carried out an open-label, uncontrolled trial and a double-blind, vehicle-controlled trial to evaluate the efficacy and safety of capsaicin $0.025 \%$ cream in the treatment of localized areas of pruritus in patients undergoing long term HD. Eight of nine evaluable patients in the open-label trial reported marked relief or complete resolution of itching during the study period, and 2 of 5 evaluable patients in the double-blind trial reported complete resolution of itching in the capsaicin-treated arm with no or minimal improvement in the vehicle-treated arm. Twelve patients in the open-label trial and two in the double-blind trial were unevaluable. No serious treatment-related adverse reactions occurred.

Further, Tarng et al. [40] reported that 19 HD patients with idiopathic, moderate $(\mathrm{n}=5)$ to severe $(\mathrm{n}=14)$ pruritus were examined in a double-blind, placebo-controlled, crossover study, and 17 of them completed the study. Topical capsaicin or placebo base cream was applied to localized areas of pruritus 4 times a day. The severity of pruritus and treatment-related side effects (cutaneous burning/stinging sensations, dryness, or erythema) were evaluated weekly. The results showed that 1) 14 of 17 patients reported marked relief, and 5 of these 14 patients had complete remission of pruritus during capsaicin treatment $(P<0.001)$; 2$)$ capsaicin was significantly more effective than placebo $(P<0.001)$, and a prolonged antipruritic effect was observed 8 weeks posttreatment; 3 ) no serious side effects were noted during the study; and 4) there were no significant changes in serum concentrations of albumin, calcium, phosphorus, alkaline phosphatase, or iPTH during the treatment with either capsaicin or placebo. According to their results, this study provides indirect evidence that in idiopathic pruritus in some patients on maintenance HD, substance P may be transmitted from the peripheral sensory neurons to the central nervous system because local application of capsaicin depletes the peripheral neurons of substance $\mathrm{P}$ and may block the conduction of pruritus. Moreover, the unique pharmacological effect of topical capsaicin may be able to markedly improve the pruritus of certain patients.

In another study, Weisshaar et al. [41] reported that 11 pruritic patients on HD and 10 controls were treated with capsaicin $0.05 \%$ liniment on the upper back three times daily for 5 days. Study parameters investigated were wheal and flare reactions, itch, and alloknesis (perifocal itch sensation induced by usually non-itching stimuli) after serotonin and histamine iontophoresis in treated and untreated skin. There were no significant differences in any parameter before and after HD. In both groups, itching was not significantly reduced by capsaicin as compared to untreated skin. Itching, however, was significantly lower in capsaicin-pretreated patients when comparing to controls. They summarized that topical capsaicin showed some antipruritic potency in HD patients in this experimental model and may therefore be considered as a co-medication in HD patients.

In addition to the components described above, essential fatty acids and their derivatives are necessary for normal cutaneous function and are thus proposed as potential treatments of pruritus. Tamimi et al. [42] found that primrose oil rich in the essential fatty acid gamma-linolenic acid (GLA) may be beneficial in alleviating pruritus, although findings did not reach statistical significance. Chen et al. [43] hypothesized that transepidermal absorption of GLA or its metabolites mediate a local anti-inflammatory and immunoregulatory effect, providing relief from pruritus. They found that GLA-rich cream was better than placebo-based cream for alleviating uremic pruritus, thus it is a useful adjuvant in the management of refractory uremic pruritus.

A new topically active antipruritic medication has been derived from the Amazonian Medicine Sangre de Grado [44]. The reported antipruritic effect on itch induced by insect bite was convincing, based on its role as a potent inhibitor of sensory afferent nerves. Moreover, Sangre de Grado is an effective analgesic and anti-inflammatory agent when applied topically.

In addition to naturally-derived agents, chemical formulations, such as Tacrolimus, have also been studied. Tacrolimus is a calcineurin inhibitor that reduces the synthesis of IL-2 by lymphocytes. Pauli-Magnus et al. [45] reported that 3 patients on PD, severely suffering from CKD-associated pruritus and previously treated, to no avail, with other potentially effective modalities, were recruited. The patients evaluated pruritus using VAS, ranging from 0 to 10, and a detailed pruritus score 3 days prior to and during the treatment phase. Patients were instructed to apply a tacrolimus ointment $0.03 \%$ twice daily, for a period of 7 days, to areas most affected by CKD-associated pruritus. In all 3 patients, CKD-associated pruritus was reduced dramatically right from the start of treatment. Two days after treatment was stopped, pruritus slowly recurred. No side effects were noted during or after the treatment period. 
Duque et al. [46] reported that in an attempt to confirm these findings, they conducted a randomized, doubleblind, vehicle-controlled study to assess the efficacy of tacrolimus ointment $0.1 \%$ for the treatment of HD-related pruritus. The results of this study did not demonstrate that tacrolimus ointment $0.1 \%$ was more effective than vehicle in relieving uremic pruritus. Topical steroids were also prescribed to these patients, probably based on the assumption that drugs used for itch in other conditions may also work on uremic pruritus, however, the absence of controlled studies and potential serious side effects of these agents dampen the routine prescription of such drugs to HD patients.

\subsection{Ultraviolet Irradiation}

Ultraviolet, especially narrowband UVB, has been proposed as a potential therapeutic agent for pruritus. Although exact mechanisms of UVB therapy in CKD-associated pruritus is unknown, some possible explanations have been proposed such as inactivation of circulating pruritogenic substances [47] [48], suppression of histamine release from cutaneous mast cells [49], and reduction of cutaneous nerve fibers [50] [51]. Blachley et al. [52] reported that 17 patients presenting with severe pruritus were treated thrice weekly with total body exposure to either UVA or UVB light. UVB light resulted in resolution of pruritus in all cases. UVA light was without any significant effect. Skin biopsies obtained before and after UV phototherapy revealed elevated contents of calcium, magnesium, and phosphorus in all pruritic patients. The resolution of pruritus following UVB treatment was associated with a reduction in skin phosphorus to values comparable with nonpruritic uremics or healthy volunteers. Uremic pruritus may be due to increased skin divalent ion content resulting in microprecipitation of calcium or magnesium phosphate. The mechanism by which UVB improves pruritus is not clear, but it has been suggested that it may in part be due to its ability to reduce cytokine production by lymphocytes.

\subsection{Acupuncture}

Acupuncture can be defined as the stimulation of anatomical points on the body using a variety of techniques for therapeutic purposes. The acupuncture technique that has been most often studied scientifically involves penetrating the skin with thin, solid, metallic needles that are manipulated by the hands or by electrical stimulation. Acupuncture has been practiced in China and other Asian countries for thousands of years, and is one of the key components of traditional medicine in the East Asia area, although it has developed differently in each region.

Recently, Kim et al. [53] reported a systemic review of acupuncture for treating uremic pruritus in patients with ESRD. According to their analysis, all of the included subjects reported beneficial effects of acupuncture. Che-yi et al. [54] randomized 40 patients with refractory uremic pruritus into two groups; in group 1 (n = 20), acupuncture was applied unilaterally at the Quchi (LI11) acupoint thrice weekly for 1 month, and in group 2 (controls, $\mathrm{n}=20$ ), acupuncture was applied at a non-acupoint $2 \mathrm{~cm}$ lateral to Quchi (LI11) thrice weekly for 1 month. Subjects responded to a pruritus score questionnaire given before and at the end of the 1 month treatment and at a 3-month follow-up. The results of the pruritus scores were analyzed with the repeated measures general linear model to examine the effect of acupuncture on pruritus scores. In their findings, pruritus scores before and after acupuncture, and at the 3-month follow-up were $38.3 \pm 4.3,17.3 \pm 5.5$, and $16.5 \pm 4.9$ in group 1 , and 38.3 \pm 4.3 , $37.5 \pm 3.2$, and $37.1 \pm 5$ in group 2 (controls), respectively. Laboratory tests showed no significant differences between the two groups. Pruritus scores were significantly lower after acupuncture and at the 3-month follow-up with a significance of $P<0.001$. From these findings, the authors concluded that acupuncture at the Quchi (LI11) acupoint is an easy, safe, and effective method of relieving uremic pruritus.

In another study by Gao et al. [55], 68 cases were randomly divided by half into two groups, acupuncture or drug administration with chlor-trimenton and topical ointment for 2 weeks. While receiving HD treatment, the acupuncture group received treatment at Quchi (LI11) with lifting-thrusting reducing method, and Zusanli (ST36) with lifting-thrusting reinforcing method for $30 \mathrm{~min}$. In patients who received acupuncture therapy, after one course of treatment, 24 of 34 cases (70.6\%) had complete alleviation of pruritus, 9 cases (26.5\%) had obvious alleviation of pruritus, and 1 case had no improvement.

Sakurada et al. [56] reported that almost one-third of HD patients had undergone acupuncture or had a desire to try acupuncture treatment to manage common complications. Similar findings were also reported by Shapiro et al. [57] in an observational study. Further, electro-acupuncture was performed on 7 HD patients with pruritus. It was noted that all the patients experienced partial or complete symptom relief during or after the electro-acupuncture treatment. Complete relief after one session of acupuncture treatment was reported in 6 patients and 
effects lasted up to one year.

There were very few undesirable side effects reported during and after acupuncture treatment. The mechanisms of action of acupuncture are of utmost interest. As described in the section on the pathophysiology of pruritus, it is likely that acupuncture may modulate pruritus through the endogenous opioids system. As pain and pruritus have similar pattern of activation, acupuncture analgesia is initiated by stimulation, in the muscles, of high-threshold small-diameter neurons. These nerves are able to send messages to the spinal cord and then activate the spinal cord, brain stem, and hypothalamic neurons, which, in turn, trigger endogenous opioid mechanisms.

Our group recently demonstrated a marked improvement of symptoms related with pruritus. Acupuncture was administered in $12 \mathrm{HD}$ patients 1 to 3 times a week for one year. With improvement of these symptoms, QOL as evaluated by short form 36 (SF-36) health survey showed a marked increase in physical activity and sleep quality (personal communication). Interestingly, the BUN levels were significantly decreased, and those of hemoglobin increased although it did not reach statistical significance (Figures 1-3).

\subsection{Rubdown with Japanese Dry Towels}

Our group examined the effects of "rubdown with Japanese dry towels" on CKD-associated pruritus. This method is a traditional Japanese alternative medical treatment to strengthen the barrier function of the skin. Briefly, subjects were naked or wore minimal clothing to maximally expose the skin of their body. Then, the subjects prepared three sets of Japanese dry towels made with cotton. These towels were cleansed with water and then dried under sunlight. After drying, the subjects gently rubbed their whole body with these towels, and if possible, this procedure was carried out in direct sunlight. The results are shown in Figure 4. The mechanism by which this traditional Japanese alternative medical procedure aids in symptom relief may be because skin-rubbing produces secretion of corticosteroid hormone through stimulation of the thalamus [58]. Further, skin-rubbing eliminates the bacterial flora on the surface of the skin [59]. In combination with ultraviolet rays, skin-rubbing may prevent intrusion of c-fiber from the dermis into the epidermis which is one of the causes of itch [20] [60].

\subsection{Opioid Antagonists}

The use of opioid antagonists in uremic pruritus was first brought to our attention by Andersen et al. [61] when they published a case report about a terminally ill uremic patient successfully treated by naloxone for persistent

Pruritus $(\mathrm{mm})$

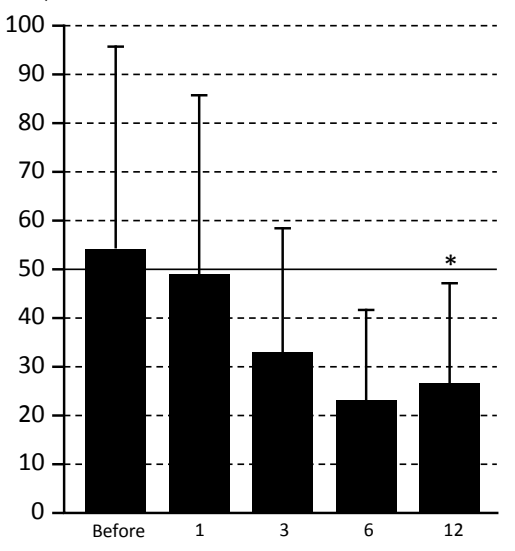

Months

\section{General symptoms}

$(\mathrm{mm})$



Figure 1. Changes in pruritus and general symptoms related with hemodialysis therapy by acupuncture 1 to 3 times a week for one year. Acupuncutre 1 to 3 times a week for one year produced a marked improvement in pruritus and general symptoms in patients with hemodialysis. * indicates $P<0.05$. 
Energy

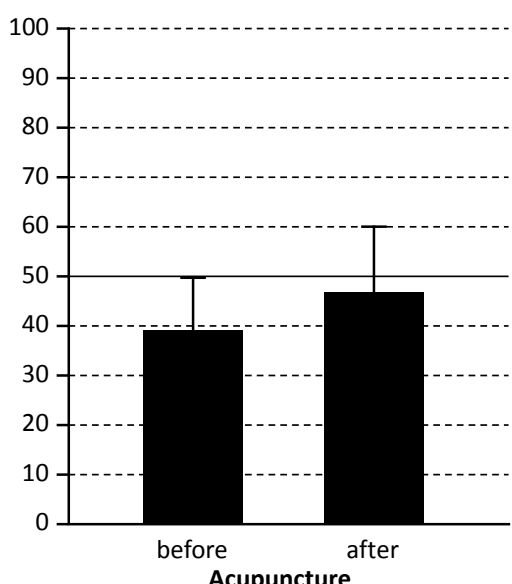

Sleep

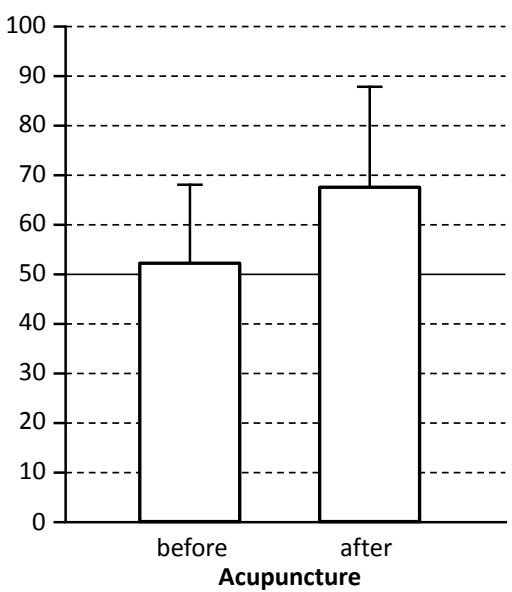

Figure 2. Changes in energy level and sleep of patients with hemodialysis by acupuncture 1 to 3 times a week for one year as evaluated by SF-36. Acupuncutre 1 to 3 times a week for one year produced a marked improvement in energy level and sleep in patients with hemodialysis.
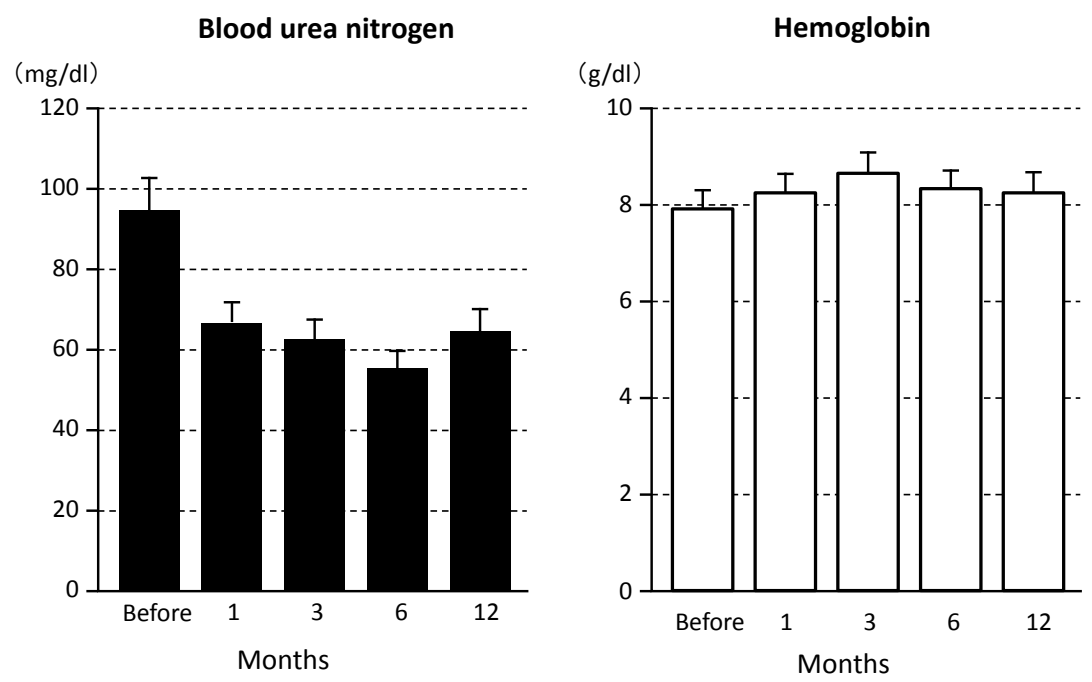

Figure 3. Changes in blood urea nitrogen and hemoglobin levels by acupuncture 1 to 3 times a week for one year. Acupuncutre 1 to 3 times a week for one year produced a marked improvement in blood urea nitrogen and hemoglobin levels in patients with hemodialysis.

itching. However, few studies were published, and these had conflicting findings. While Peer et al. [62] showed in a small placebo-controlled clinical trial that naltrexone, which is a $\mu$-receptor antagonist, is effective, PauliMagnus et al. [45] failed to demonstrate any efficacy of naltrexone in the treatment of uremic pruritus. Later, Legroux-Crespel et al. [63] conducted a comparative study between naltrexone and loratadine, and concluded that naltrexone is not effective and not well-tolerated because of frequent side effects, except in a small subset of patients. More recently, another perspective was elaborated regarding the use of a $k$-agonist, for $k$-receptor stimulation inhibits $\mu$-receptor effects both peripherally and centrally, and hence might inhibit itching induced by substance P. In line with this concept, Wikstrom et al. [64] conducted two multicenter, randomized, double-blind, placebo-controlled studies that enrolled 144 patients with uremic pruritus to receive post-dialysis intravenous treatment with either nalfurafine, a novel $k$-receptor agonist, or placebo for 2 to 4 weeks. Statistically significant reductions in itching, itching intensity, excoriations, and sleep disturbances were noted in the nalfurafine group as compared to the placebo group. 
In light of all these findings, Toray Industries, Inc., Japan, recently developed nalfurafine, with refined opioid receptor affinity and selectivity, as an agent for relief of pruritus [65] [66]. In studies using animal models, nalfurafine exerted antipruritic activity not only for antihistamine-sensitive itch, but also for antihistamine-resistant itch [67] [68].

Kumagai et al. [69] carried out a prospective, randomized, double-blind comparative study for 2 weeks to compare the antipruritic effect of oral nalfurafine ( 2.5 and $5.0 \mu \mathrm{g})$ with a placebo in 337 patients. The mean pruritus value as assessed by VAS was $75.2 \mathrm{~mm}$ during the pre-observation period, which decreased significantly to 50.9 in weeks 2 . The mean decrease in VAS from baseline was significantly larger in the $2 \mu \mathrm{g}(\mathrm{n}=112, P=$ $0.0001)$ and $5 \mu \mathrm{g}(\mathrm{n}=114, P=0.0002)$ nalfurafine groups than in the placebo group $(\mathrm{n}=111)$. However, adverse drug reactions (ADRs) occurred in 103 patients, and the incidence was $25.0 \%$ in the $2.5 \mu$ g group, 35.1\% in the $5 \mu$ group, and $16.2 \%$ in the placebo group. The most common ADR was insomnia, observed in 24 of the 226 nalfurafine patients (22.3\%). It is interesting to note that the group that received placebo also had a similar decrease in itching. It is well known that placebo-induced expectancies have been shown to decrease pain in a manner reversible by opioid antagonists. This phenomenon is corroborated by the findings of Wager et al. [70], who demonstrated using fMRI that placebo analgesia was related to decreased brain activity in pain-sensitive brain regions, including the thalamus, insula, and anterior cingulate cortex.

Further, Kumagai et al. [71] carried out an open-label study examining the effects and ADRs of 52-week oral administration of nalfurafine hydrochloride in 211 HD patients with treatment-resistant itch. They found that the mean pruritus values as assessed by the VAS was $75.2 \mathrm{~mm}$ during the pre-observation period, which decreased significantly to 50.9 and $30.9 \mathrm{~mm}$ in weeks 52, indicating a long-lasting efficacy. ADRs occurred in 103 patients. Frequent ADRs were insomnia (19.4\%), constipation (7.1\%), and increased blood prolactin (3.3\%).

\section{Advantages and Disadvantages in Modalities for Treatment for Hemodialyzed Patients with Pruritus}

The modalities discussed in this review are summarized in the Table 1.

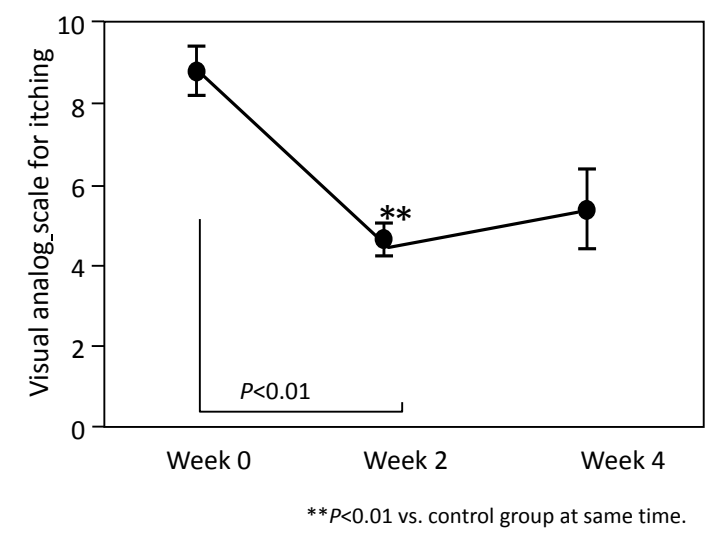

Figure 4. Effect of rubdown with Japanese dry towels on change in visual analog scale for itching. Manipulation by rubdown with Japanese dry towels produced a marked reduction in pruritus using the visual analog scale. $* *$ indicates $P<0.01$.

Table 1. Comparison of various modalities for treatment for hemodialyzed patients with pruritus.

\begin{tabular}{ccc}
\hline Modality & Advantages & Disadvantages \\
\hline Modification of dialysis techniques & Easy & Not always effective \\
Emollients & Use for first-line treatment & Needs a large amount \\
Ultraviolet irradiation & Effective & Unclear for mechanisms \\
Acupuncture & Safe and effective & Needs technique \\
Rubdown with Japanese dry towels & Easy and safe & Not always effective \\
Opioid antagonists & Effective & Expensive \\
\hline
\end{tabular}




\section{Future Perspectives}

As stated in this review, recent advances in pathophysiology of itch and treatment for CKD-associated pruritus have improved this condition remarkably, however, there are still a lot of obstacles to overcome in order to achieve satisfactory comfort and relief from unpleasant symptoms stemming from pruritus. It is therefore of the utmost importance for investigators and physicians to study and research in this area.

\section{References}

[1] Stahle-Backdahl, M. (1989) Uremic Pruritus. Clinical and Experimental Studies. Acta Dermato-Venereologica. Supplementum, 145, 1-38.

[2] Stahle-Backdahl, M. (1992) Pruritus in Hemodialysis Patients. Skin Pharmacology and Physiology, 5, 14-20. http://dx.doi.org/10.1159/000211011

[3] Stahle-Backdahl, M. (1995) Uremic Pruritus. Seminars in Dermatology, 14, 297-301. http://dx.doi.org/10.1016/S1085-5629(05)80051-3

[4] Manenti, L., Tansinda, P. and Vaglio, A. (2009) Uraemic Pruritus: Clinical Characteristics, Pathophysiology and Treatment. Drugs, 69, 251-263. http://dx.doi.org/10.2165/00003495-200969030-00002

[5] Yosipovitch, G., Greaves, M.W. and Schmelz, M. (2003) Itch. The Lancet, 361, 690-694. http://dx.doi.org/10.1016/S0140-6736(03)12570-6

[6] Lugon, J.R. (2005) Uremic Pruritus: A Review. Hemodialysis International, 9, 180-188. http://dx.doi.org/10.1111/j.1492-7535.2005.01130.x

[7] Kosmadakis, G.C. and Zerefos, N. (2006) Uremic Pruritus. The International Journal of Artificial Organs, 29, 938-943.

[8] Greaves, M.W. (2007) Recent Advances in Pathophysiology and Current Management of Itch. Annals Academy of Medicine Singapore, 36, 788-792.

[9] Patel, T.S., Freedman, B.I. and Yosipovitch, G. (2007) An Update on Pruritus Associated with CKD. American Journal of Kidney Diseases, 50, 11-20. http://dx.doi.org/10.1053/j.ajkd.2007.03.010

[10] Narita, I., Iguchi, S., Omori, K. and Gejyo, F. (2008) Uremic Pruritus in Chronic Hemodialysis Patients. Journal of Nephrology, 21, 161-165.

[11] Berger, T.G. and Steinhoff, M. (2011) Pruritus and Renal Failure. Seminars in Cutaneous Medicine and Surgery, 30, 99-100. http://dx.doi.org/10.1016/j.sder.2011.04.005

[12] Greaves, M.W. (2010) Pathogenesis and Treatment of Pruritus. Current Allergy and Asthma Reports, 10, $236-242$. http://dx.doi.org/10.1007/s11882-010-0117-z

[13] Kfoury, L.W. and Jurdi, M.A. (2012) Uremic Pruritus. Journal of Nephrology, 25, 644-652. http://dx.doi.org/10.5301/jn.5000039

[14] Mettang, T. and Kremer, A.E. (2014) Uremic Pruritus. Kidney International. [Equib ahead of print] http://dx.doi.org/10.1038/ki.2013.454

[15] Pontremoli, R., Sofia, A., Ravera, M., Nicolella, C., Viazzi, F., Tirotta, A., et al. (1997) Prevalence and Clinical Correlates of Microalbuminuria in Essential Hypertension: The MAGIC Study. Hypertension, 30, 1135-1143. http://dx.doi.org/10.1161/01.HYP.30.5.1135

[16] Narita, I., Alchi, B., Omori, K., Sato, F., Ajiro, J., Saga, D., et al. (2006) Etiology and Prognostic Significance of Severe Uremic Pruritus in Chronic Hemodialysis Patients. Kidney International, 69, 1626-1632. http://dx.doi.org/10.1038/sj.ki.5000251

[17] Pisoni, R.L., Wikstrom, B., Elder, S.J., Akizawa, T., Asano, Y., Keen, M.L., et al. (2006) Pruritus in Haemodialysis Patients: International Results from the Dialysis Outcomes and Practice Patterns Study (DOPPS). Nephrology Dialysis Transplantation, 21, 3495-3505. http://dx.doi.org/10.1093/ndt/gfl461

[18] Gilchrest, B.A., Stern, R.S., Steinman, T.I., Brown, R.S., Arndt, K.A. and Anderson, W.W. (1982) Clinical Features of Pruritus among Patients Undergoing Maintenance Hemodialysis. JAMA Dermatology, 118, 154-156. http://dx.doi.org/10.1001/archderm.1982.01650150016012

[19] Dar, N.R. and Akhter, A. (2006) Clinical Characteristics of Uremic Pruritus in Patients Undergoing Haemodialysis. Journal of the College of Physicians and Surgeons-Pakistan, 16, 94-96.

[20] Tominaga, M. and Takamori, K. (2014) Sensitization of Itch Signaling: Itch Sensitization—Nerve Growth Factor, Semaphorins.

[21] Kumagai, H., Matsukawa, S., Sasamura, H., Hayashi, M. and Saruta, T. (2004) Prospects for a Novel к-Opioid Recep- 
tor Agonist, TRK-820, in Uremic Pruritus. In: Yoshipovitch, G., Ed., Itch Basic Mechanisms and Therapy, Marcel Dekker, New York, 279-286.

[22] Walter, B., Sadlo, M.N., Kupfer, J., Niemeier, V., Brosig, B., Stark, R., Vaitl, D. and Gieler, U. (2005) Brain Activation by Histamine Prick Test-Induced Itch. Journal of Investigative Dermatology, 125, 380-382.

[23] Schmelz, M., Hilliges, M., Schmidt, R., Ørstavik, K., Vahlquist, C., Weidner, C., et al. (2003) Active "Itch Fibers” in Chronic Pruritus. Neurology, 61, 564-566. http://dx.doi.org/10.1212/01.WNL.0000078193.64949.08

[24] Liakopoulos, V., Krishnan, M., Stefanidis, I., Savaj, S., Ghareeb, S., Musso, C., et al. (2004) Improvement in Uremic Symptoms after Increasing Daily Dialysate Volume in Patients on Chronic Peritoneal Dialysis with Declining Renal Function. International Urology and Nephrology, 36, 437-443. http://dx.doi.org/10.1007/s11255-004-8788-9

[25] Szepietowski, J.C., Reich, A. and Szepietowski, T. (2005) Emollients with Endocannabinoids in the Treatment of Uremic Pruritus: Discussion of the Therapeutic Options. Therapeutic Apheresis and Dialysis, 9, 277-279. http://dx.doi.org/10.1111/j.1774-9987.2005.00271.x

[26] Novak, M.J., Sheth, H., Bender, F.H., Fried, L. and Piraino, B. (2008) Improvement in Pittsburgh Symptom Score Index after Initiation of Peritoneal Dialysis. Advances in Peritoneal Dialysis, 24, 46-50.

[27] Hiroshige, K., Kabashima, N., Takasugi, M. and Kuroiwa, A. (1995) Optimal Dialysis Improves Uremic Pruritus. American Journal of Kidney Diseases, 25, 413-419. http://dx.doi.org/10.1016/0272-6386(95)90102-7

[28] Graf, H., Kovarik, J., Stummvoll, H.K. and Wolf, A. (1979) Disappearance of Uraemic Pruritus after Lowering Dialysate Magnesium Concentration. British Medical Journal, 2, 1478-1479. http://dx.doi.org/10.1136/bmj.2.6203.1478-a

[29] Carmichael, A.J., Dickinson, F., McHugh, M.I., Martin, A.M. and Farrow, M. (1988) Magnesium Free Dialysis for Uraemic Pruritus. British Medical Journal, 297, 1584-1585. http://dx.doi.org/10.1136/bmj.297.6663.1584

[30] Kyriazis, J. and Glotsos, J. (2000) Dialysate Calcium Concentration of $\leq 1.25 \mathrm{mmol} / \mathrm{l}$ : Is It Effective in Suppressing Uremic Pruritus? Nephron, 84, 85-86. http://dx.doi.org/10.1159/000045546

[31] Kato, A., Takita, T., Furuhashi, M., Takahashi, T., Watanabe, T., Maruyama, Y. and Hishida, A. (2001) Polymethylmethacrylate Efficacy in Reduction of Renal Itching in Hemodialysis Patients: Crossover Study and Role of Tumor Necrosis Factor-Alpha. Artificial Organs, 25, 441-447. http://dx.doi.org/10.1046/j.1525-1594.2001.025006441.x

[32] Morton, C.A., Lafferty, M., Hau, C., Henderson, I., Jones, M. and Lowe, J.G. (1996) Pruritus and Skin Hydration during Dialysis. Nephrology Dialysis Transplantation, 11, 2031-2036.

http://dx.doi.org/10.1093/oxfordjournals.ndt.a027092

[33] Twycross, R., Greaves, M.W., Handwerker, H., Jones, E.A., Libretto, S.E., Szepietowski, J.C. and Zylicz, Z. (2003) Itch: Scratching More than the Surface. QJM: An International Journal of Medicine, 96, 7-26. http://dx.doi.org/10.1093/qjmed/hcg002

[34] Szepietowski, J.C., Szepietowski, T. and Reich, A. (2005) Efficacy and Tolerance of the Cream Containing Structured Physiological Lipids with Endocannabinoids in the Treatment of Uremic Pruritus: A Preliminary Study. Acta Dermatovenerologica Croatica, 13, 97-103.

[35] Okada, K. and Matsumoto, K. (2004) Effect of Skin Care with an Emollient Containing a High Water Content on Mild Uremic Pruritus. Therapeutic Apheresis and Dialysis, 8, 419-422. http://dx.doi.org/10.1111/j.1526-0968.2004.00175.x

[36] Itai, T., Amayasu, H., Kuribayashi, M., Kawamura, N., Okada, M., Momose, A., et al. (2000) Psychological Effects of Aromatherapy on Chronic Hemodialysis Patients. Psychiatry and Clinical Neurosciences, 54, 393-397. http://dx.doi.org/10.1046/j.1440-1819.2000.00727.x

[37] Dvorak, M., Watkinson, A., McGlone, F. and Rukwied, R. (2003) Histamine Induced Responses Are Attenuated by a Cannabinoid Receptor Agonist in Human Skin. Inflammation Research, 52, 238-245.

[38] Facci, L., Dal Toso, R., Romanello, S., Buriani, A., Skaper, S.D. and Leon, A. (1995) Mast Cells Express a Peripheral Cannabinoid Receptor with Differential Sensitivity to Anandamide and Palmitoylethanolamide. Proceedings of the National Academy of Sciences of the United States of America, 92, 3376-3380. http://dx.doi.org/10.1073/pnas.92.8.3376

[39] Breneman, D.L., Cardone, J.S., Blumsack, R.F., Lather, R.M., Searle, E.A. and Pollack, V.E. (1992) Topical Capsaicin for Treatment of Hemodialysis-Related Pruritus. Journal of the American Academy of Dermatology, 26, 91-94. http://dx.doi.org/10.1016/0190-9622(92)70013-6

[40] Tarng, D.C., Cho, Y.L., Liu, H.N. and Huang, T.P. (1996) Hemodialysis-Related Pruritus: A Double-Blind, PlaceboControlled, Crossover Study of Capsaicin 0.025\% Cream. Nephron, 72, 617-622. http://dx.doi.org/10.1159/000188949

[41] Weisshaar, E., Dunker, N. and Gollnick, H. (2003) Topical Capsaicin Therapy in Humans with Hemodialysis-Related Pruritus. Neuroscience Letters, 345, 192-194. http://dx.doi.org/10.1016/S0304-3940(03)00511-1

[42] Tamimi, N.A., Mikhail, A.I. and Stevens, P.E. (1999) Role of $\gamma$-Linolenic Acid in Uraemic Pruritus. Nephron, 83, 170171. http://dx.doi.org/10.1159/000045498 
[43] Chen, Y.C., Chiu, W.T. and Wu, M.S. (2006) Therapeutic Effect of Topical Gamma-Linolenic Acid on Refractory Uremic Pruritus. American Journal of Kidney Diseases, 48, 69-76. http://dx.doi.org/10.1053/j.ajkd.2006.03.082

[44] Miller, M.J.S., Vergnolle, N., McKnight, W., Musah, R.A., Davison, C.A., Trentacosti, A.M., et al. (2001) Inhibition of Neurogenic Inflammation by the Amazonian Herbal Medicine Sangre de Grado. Journal of Investigative Dermatology, 117, 725-730.

[45] Pauli-Magnus, C., Mikus, G., Alscher, D.M., Kirschner, T., Nagel, W., Gugeler, N., et al. (2000) Naltrexone Does Not Relieve Uremic Pruritus: Results of a Randomized, Double-Blind, Placebo-Controlled Crossover Study. Journal of the American Society of Nephrology, 11, 514-519.

[46] Duque, M.I., Thevarajah, S., Chan, Y.H., Tuttle, A.B., Freedman, B.I. and Yosipovitch, G. (2006) Uremic Pruritus Is Associated with Higher kt/V and Serum Calcium Concentration. Clinical Nephrology, 66, 184-191. http://dx.doi.org/10.5414/CNP66184

[47] Gilchrest, B.A., Rowe, J.W., Brown, R.S., Steinman, T.I. and Arndt, K.A. (1979) Ultraviolet Phototherapy of Uremic Pruritus: Long-Term Results and Possible Mechanism of Action. Annals of Internal Medicine, 91, 17-21. http://dx.doi.org/10.7326/0003-4819-91-1-17

[48] Shultz, B. and Roenigk, H. (1980) Uremic Pruritus Treated with Ultraviolet Light. Journal of American Medical Association, 243, 1836-1837. http://dx.doi.org/10.1001/jama.1980.03300440038023

[49] Imazu, L.E., Tachibana, T., Danno, K., Tanaka, M. and Imamura, S. (1993) Histamine-Releasing Factor(s) in Sera of Uraemic Pruritus Patients in a Possible Mechanism of UVB Therapy. Archives of Dermatological Research, 285, 423427. http://dx.doi.org/10.1007/BF00372137

[50] Fjellner, B. and Hagermark, O. (1982) Influence of Ultraviolet Light on Itch and Flare Reactions in Human Skin Induced by Histamine and the Histamine Liberator Compound 48/80. Acta Dermato-Venereologica, 62, 137-140.

[51] Wallengren, J. and Sundler, F. (2004) Phototherapy Reduces the Number of Epidermal and CGRP-Positive Dermal Nerve Fibres. Acta Dermato-Venereologica, 84, 111-115. http://dx.doi.org/10.1080/00015550310022899

[52] Blachley, J.D., Blankenship, D.M., Menter, A., Parker III, T.F. and Knochel, J.P. (1985) Uremic Pruritus: Skin Divalent Ion Content and Response to Ultraviolet Phototherapy. American Journal of Kidney Diseases, 5, 237-241. http://dx.doi.org/10.1016/S0272-6386(85)80115-3

[53] Kim, K.H., Lee, M.S., Choi, S.M. and Ernst, E. (2010) Acupuncture for Treating Uremic Pruritus in Patients with End-Stage Renal Disease: A Systematic Review. Journal of Pain and Symptom Management, 40, 117-125. http://dx.doi.org/10.1016/j.jpainsymman.2009.11.325

[54] Che-Yi, C., Wen, C.Y., Min-Tsung, K. and Chiu-Ching, H. (2005) Acupuncture in Haemodialysis Patients at the Quchi (LI11) Acupoint for Refractory Uraemic Pruritus. Nephrology Dialysis Transplantation, 20, 1912-1915. http://dx.doi.org/10.1093/ndt/gfh955

[55] Gao, H., Zhang, W. and Wang, Y. (2002) Acupuncture Treatment for 34 Cases of Uremic Cutaneous Pruritus. Journal of Traditional Chinese Medicine, 22, 29-30.

[56] Sakuraba, H., Takeuchi, H., Takeuchi, M., Syoji, M. and Moriyama, T. (2007) Questionnaire Survey of Complaints and Accupuncutre Treatment in Maintenance Hemodialysis Patients. Nihon Toseki Igakkai Zasshi, 40, 513-516. http://dx.doi.org/10.4009/jsdt.40.513

[57] Shapiro, R., Stockard, H. and Schank, A. (1986) Successful Treatment of Uremic Pruritus with Acupuncutre. American Journal of Acupuncture, 14, 235-242.

[58] Antoni, F. (1986) Hypothalamic Control of Adrenocorticotropin Secretion: Advances Since the Discovery of 41-Redue Corticotropin-Releasing Factor. Endocrine Reviews, 7, 351-378. http://dx.doi.org/10.1210/edrv-7-4-351

[59] Percival, S.L., Emanuel, C., Cutting, K.F. and Williams, D.W. (2012) Microbiology of the Skin and the Role of Biofilms in Infection. International Wound Journal, 9, 14-32. http://dx.doi.org/10.1111/j.1742-481X.2011.00836.x

[60] Tominaga, M. and Takamori, K. (2013) An Update on Peripheral Mechanisms and Treatments of Itch. Biological and Pharmaceutical Bulletin, 36, 1241-1247. http://dx.doi.org/10.1248/bpb.b13-00319

[61] Andersen, L.W., Friedberg, M. and Lokkegaard, N. (1984) Naloxone in the Treatment of Uremic Pruritus: A Case History. Clinical Nephrology, 21, 355-356.

[62] Peer, G., Kivity, S., Agami, O., Fireman, E., Silverberg, D., Blum, M. and Iaina, A. (1996) Randomised Crossover Trial of Naltrexone in Uraemic Pruritus. Lancet, 348, 1552-1554. http://dx.doi.org/10.1016/S0140-6736(96)04176-1

[63] Legroux-Crespel, E., Cledes, J. and Misery, L. (2004) A Comparative Study on the Effects of Naltrexone and Loratadine on Uremic Pruritus. Dermatology, 208, 326-330. http://dx.doi.org/10.1159/000077841

[64] Wikstrom, B., Gellert, R., Ladefoged, S.D., Danda, Y., Akai, M., Ide, K., et al. (2005) к-Opioid System in Uremic Pruritus: Multicenter, Randomized, Double-Blind, Placebo-Controlled Clinical Studies. Journal of the American Society of Nephrology, 16, 3742-3747. http://dx.doi.org/10.1681/ASN.2005020152 
[65] Seki, T., Awamura, S., Kimura, C., Ide, S., Sakano, K., Minami, M., et al. (1999) Pharmacological Properties of TRK-820 on Cloned $\mu$-, $\delta$ - and $\kappa$-Opioid Receptors and Nociceptin Receptor. European Journal of Pharmacology, 376, 159-167. http://dx.doi.org/10.1016/S0014-2999(99)00369-6

[66] Nagase, H., Hayakawa, J., Kawamura, K., Kawai, K., Takezawa, Y., Matsuura, H., et al. (1998) Discovery of a Structurally Novel Opioid $\kappa$-Agonist Derived from 4,5-Epoxymorphinan. Chemical and Pharmaceutical Bulletin (Tokyo), 46, 366-369. http://dx.doi.org/10.1248/cpb.46.366

[67] Wakasa, Y., Fujiwara, A., Umeuchi, H., Endoh, T., Okano, K., Tanaka, T. and Nagase, H. (2004) Inhibitory Effects of TRK-820 on Systemic Skin Scratching Induced by Morphine in Rhesus Monkeys. Life Sciences, 75, 2947-2957. http://dx.doi.org/10.1016/j.lfs.2004.05.033

[68] Togashi, Y., Umeuchi, H., Okano, K., Ando, N., Yoshizawa, Y., Honda, T., et al. (2002) Antipruritic Activity of the к-Opioid Receptor Agonist, TRK-820. European Journal of Pharmacology, 435, 259-264. http://dx.doi.org/10.1016/S0014-2999(01)01588-6

[69] Kumagai, H., Ebata, T., Takamori, K., Muramatsu, T., Nakamoto, H. and Suzuki, H. (2010) Effect of a Novel к-Receptor Agonist, Nalfurafine Hydrochloride, on Severe Itch in 337 Haemodialysis Patients: A Phase III, Randomized, Double-Blind, Placebo-Controlled Study. Nephrology Dialysis Transplantation, 25, 1251-1257. http://dx.doi.org/10.1093/ndt/gfp588

[70] Wager, T.D., Rilling, J.K., Smith, E.E., Sokolik, A., Casey, K.L., Davidson, R.J., et al. (2004) Placebo-Induced Changes in FMRI in the Anticipation and Experience of Pain. Science, 303, 1162-1167. http://dx.doi.org/10.1126/science.1093065

[71] Kumagai, H., Ebata, T., Takamori, K., Miyasato, K., Muramatsu, T., Nakamoto, H., et al. (2012) Efficacy and Safety of a Novel $\kappa$-Agonist for Managing Intractable Pruritus in Dialysis Patients. American Journal of Nephrology, 36, 175-183. http://dx.doi.org/10.1159/000341268 